\title{
EAS 3D triangular and quadrangular shell elements
}

\author{
Philippe Jetteur — Philippe Pasquet
}

\author{
Samtech \\ Rue des Chasseurs Ardennais 8 \\ B-4031 Liège, Belgium \\ \{Philippe.Jetteur, Philippe.Pasquet\}@samtech.com
}

\begin{abstract}
A new 3D solid shell element is developed in SAMCEF ${ }^{\mathrm{TM}}$ code. The purpose of this element is to make the meshing easier starting from a $3 D$ definition of the structure, it is not necessary to extract the mean surface of the shell. Here, we are not concerned by the meshing; we only are concerned by the element formulation. In order to improve the quality of the results, we add internal degrees of freedom as suggested by Simo and co-authors. We use the Enhanced Assumed Strain method. A special handling of the transverse shear is performed in order to pass successfully the plate patch test (constant bending) and to avoid shear locking. The formulation is based on the work of Bathe and Dvorkin for classical shell. The element has been developed in linear and non-linear analysis; it can be a mono or multilayer element.

RÉSUMÉ. Un nouvel élément de coque $3 D$ a été développé dans SAMCEFTM. Le but initial est de simplifier le maillage en partant directement d'une géométrie $3 D$ sans passer par la case "extraction de la surface moyenne ". Mais au-delà, il faut aussi assurer une formulation robuste basée sur des aspects théoriques et sur une étape de validation. L'élément est donc de forme hexaédrique à 8 næuds. Pour améliorer la qualité des résultats, nous ajoutons des degrés de libertés (ddl) internes proposés par Simo. Nous utilisons la méthode Enhanced Assumed Strain. Un traitement particulier des termes de cisaillement permet de passer avec succès le patch test. La formulation est basée sur les travaux de Bathe et Dvorkin pour les coques classiques. L'élément est développé pour les analyses linéaires et non linéaires. Il peut être utilisé en configuration mono ou multicouche.
\end{abstract}

KEYWORDS: shell, EAS element, buckling

MOTS-CLÉS : coque, élément EAS, flambement.

DOI:10.3166/EJCM.19.205-215 (C) 2010 Lavoisier, Paris

EJCM - 19/2010. Giens 2009, pages 205 to 215 


\section{Introduction}

In this paper, we describe a 3D solid shell element. By 3D solid shell element, it means that the topology of the element is a brick. The element has been developed for meshing purpose. The element is based on EAS (Enhanced Assumed Strain) formulation (Simo et al., 1993) and special treatment is used for the transverse shear (Dvorkin et al., 1984). We present different versions of the element, starting from the simple one and showing the limitations of the first versions. We detail how is performed the integration through the thickness in order to keep good performance when the element is used for composite structure with several layers on the thickness.

A quadrangular and a triangular version of the element have been developed. They are first order element. They are introduced in Samcef ${ }^{\mathrm{TM}}$ software.

\section{EAS formulation}

In this section, we recall the principle of the EAS formulation (Simo et al., 1993). The starting point is a three-field variational principle. The three fields are the deformed position $(x)$, the displacement gradient $(\widetilde{F})$ and the PK1 stresses $\widetilde{P}$ :

$$
\Pi(x, \widetilde{F}, \widetilde{P})=\int\left[W(\widetilde{F})+\widetilde{P}(\operatorname{grad} x-\widetilde{F}) \mid d V+\Pi_{e x t}\right.
$$

The last term is linked to the external forces. The displacement gradient is splitted in a part which depends on the displacements and an enhanced part:

$$
\widetilde{F}=F_{c o m}+F_{e n h}=\operatorname{grad} x+F_{\text {enh }}
$$

We assumed $\widetilde{P}$ constant over the element volume and $F_{\text {enh }}$ such as $\int F_{\text {enh }} d V=0$ (orthogonality condition). The potential becomes equal to:

$$
\Pi(x, \widetilde{F}, \widetilde{P})=\int\lfloor W(\widetilde{F})\rfloor d V+\Pi_{e x t}
$$

Now, we have to define what is taken for $F_{e n h}$. We use:

$$
\begin{aligned}
& F_{\text {enh }}=F_{0} F^{\prime}(\xi, \eta, \delta) \quad F_{0}=\left.F_{c o m}\right|_{\xi=\eta=\delta=0} \\
& F^{\prime}=j_{0} / j J_{0} F^{\prime \prime}(\xi, \eta, \delta) J_{0}^{-1} \quad j_{0}=\operatorname{det}\left(J_{0}\right)
\end{aligned}
$$

$F^{\prime \prime}$ is defined in the isoparametric space, $J_{0}$ is the jacobian matrix at the centre of the element. If the integral of $F$ ', over the element volume is equal to 0 , the orthogonality condition will be fulfilled. $F^{\prime \prime}$ is chosen in such a way to have a uniform polynomial definition for $\widetilde{F}$. With the first nine terms of $\alpha$, volumetric locking is removed. With the last terms, shear locking is removed. We use:

$$
F^{\prime \prime}=\left|\begin{array}{ccc}
\alpha_{1} \xi+\alpha_{4} \xi \eta+\alpha_{7} \xi \delta & \alpha_{13} \eta+\alpha_{19} \eta \xi+\alpha_{22} \eta \delta & \alpha_{14} \delta+\alpha_{20} \delta \xi+\alpha_{23} \delta \eta \\
\alpha_{10} \xi+\alpha_{16} \xi \delta & \alpha_{2} \eta+\alpha_{5} \eta \xi+\alpha_{8} \eta \delta & \alpha_{15} \delta+\alpha_{21} \delta \xi+\alpha_{24} \delta \eta \\
\alpha_{11} \xi+\alpha_{17} \xi \eta & \alpha_{12} \eta+\alpha_{18} \eta \xi & \alpha_{3} \delta+\alpha_{6} \delta \xi+\alpha_{9} \delta \eta
\end{array}\right|
$$




\section{Shell element, version 1}

In the first version of the element, we do not introduce any shell assumption. The element is identical to a volume one with EAS formulation. The advantage is that three dimensional constitutive laws can be used. A Gaussian quadrature with $2 * 2 * 2$ points is used.

As example, we study a simply supported square plate subjected to a transversal pressure. The length is taken equal to $400 \mathrm{~mm}$, the thickness to $2 \mathrm{~mm}$, the Young modulus to $210000 \mathrm{MPa}$ and the Poisson coefficient to 0.3 . We perform a convergence study using the standard volume element and the volume with EAS formulation. Only a quarter of the plate is modelized. Table 1 gives the ratio between the numerical displacement at the centre of the plate and the theoretical one. As can be seen from the results, thanks to the enhanced strains ( $\varepsilon_{z}$ is linear over the thickness of the element), we converge towards the correct solution, even with a Poisson coefficient which is not equal to 0 . This is not the case with the standard volume. We also see that the enhanced strains remove the shear locking.

Table 1. Square plate under pressure, convergence study

\begin{tabular}{|ccc|}
\hline $\mathrm{n}$ & Standard volume & EAS Volume \\
\hline 2 & & 0.989 \\
\hline 8 & 0.035 & 1.000 \\
\hline 32 & 0.372 & 1.001 \\
\hline
\end{tabular}

We see that EAS formulation improves the convergence of the element. For $n=8$, we change the thickness of the element. Table 2 gives results for the EAS element in function of the ratio length over thickness.

Table 2. Square plate under pressure, influence of ratio length/thickness, version 1

\begin{tabular}{|c|c|}
\hline$L / t$ & EAS Volume \\
\hline 200 & 1.000 \\
\hline 2000 & 1.000 \\
\hline 20000 & $*$ \\
\hline
\end{tabular}

When the ratio length over thickness becomes too high, numerical problems arise. For this reason, we introduce version 2 of the element.

\section{Shell element, version 2}

In order to understand what happens when the ratio length over thickness increases, we look at a $2 \mathrm{D}$ case and we compute the order of magnitude of the 
stiffness matrix linked to one node. We start from the energy, define the strain in function of the displacement of the first element node.

$$
\begin{aligned}
& U=\frac{1}{2} \int\left[E_{x} \varepsilon_{x}^{2}+E_{z} \varepsilon_{z}^{2}+G \gamma^{2}\right] d A \\
& \varepsilon_{x}=u_{1} / 2 L \\
& \varepsilon_{z}=w_{1} / 2 t \\
& \gamma=u_{1} / 2 t+w_{1} / 2 L \\
& K=\frac{L t}{4 L^{2}}\left|\begin{array}{cc}
E_{x}+\alpha^{2} G & \alpha G \\
\alpha G & G+\alpha^{2} E_{z}
\end{array}\right| \quad \alpha=\frac{L}{t}
\end{aligned}
$$

We see that some terms are proportional to $\alpha^{2}$ and will become very large when the thickness decreases. $K_{22}$ is linked to the transversal direction. The solution we adopt is to use plane stress assumption in the shell element and to modify the transverse Young modulus, it is divided by $\max \left(1, \alpha^{2}\right)$. This modification has no influence on the results as the stress in the transverse direction is, at least, one order of magnitude smaller than the in-plane stress. Neglecting the stress in the $3^{\text {rd }}$ direction is a classical shell assumption. We also assume than the shear strains linked to transverse shear and transverse strain are constant over the shell thickness. The number of EAS terms is reduced to 12:

$$
F^{\prime \prime}=\left|\begin{array}{lll}
\alpha_{1} \xi+\alpha_{7} \xi \delta & \alpha_{4} \eta+\alpha_{10} \eta \delta & 0 \\
\alpha_{2} \xi+\alpha_{8} \xi \delta & \alpha_{5} \eta+\alpha_{11} \eta \delta & 0 \\
\alpha_{3} \xi+\alpha_{9} \xi \eta & \alpha_{6} \eta+\alpha_{12} \eta \xi & 0
\end{array}\right|
$$

We keep the same number of integration points $(2 * 2 * 2$ points) as in version 1 .

We analyse the square plate with this element. We use $8 * 8$ element meshes. Different ratios - length over thickness - are used. Table 3 gives the ration between numerical and theoretical maximum displacement.

Table 3. Square plate under pressure, influence of ratio length/thickness, version 2

\begin{tabular}{|c|c|c|}
\hline$L / t$ & Shell version 2 & Classical 4 node shell element \\
\hline 200 & 1.000 & 1.000 \\
\hline 2000 & 1.000 & 1.000 \\
\hline 20000 & 1.000 & 1.000 \\
\hline 200000 & 1.000 & 1.000 \\
\hline 2000000 & $*$ & 1.000 \\
\hline
\end{tabular}

The shell can be 100 times thinner than with version 1 .

We now perform a patch test analysis. The mesh we used is shown on Figure 1. 


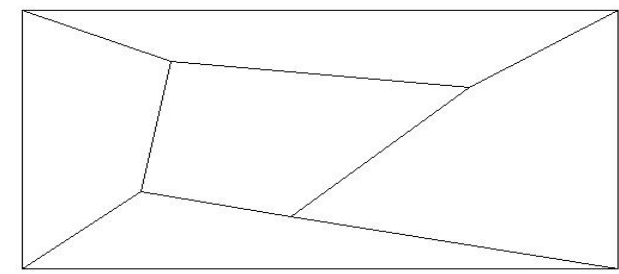

Figure 1. Patch test analysis

For a membrane loading, there is no problem, the state of stress is constant over the plate. For a bending loading, the state of stress is no more constant. For this reason, we introduce version 3 of the element. It means that a distorted element does not give good results in flexion, this is also true with the first version of the element. So even if the shell is thick, we do not think that the first version of the element should be used for a distorted mesh.

\section{Shell element, version 3}

The problem with the bending patch test comes from the transverse shear behaviour. In order to improve the element, we use the following procedure (Dvorkin et al., 1984):

- in the covariant bases, the 2 first axes are parallel to the intrinsic coordinates, the $3^{\text {rd }}$ one is parallel to the normal

- we compute transverse shear strain $\gamma_{\xi 3} \gamma_{\eta_{3}}$ at the middle of the edges, parallel to the edge

- we perform a surface interpolation of these shear strains

- we transform the strain from the covariant bases to the Cartesian one parallel to the shell surface

With respect to version 2, only the transverse shear strains are modified. Now, the bending patch test can be passed without any problem.

We build a triangular element with the same principle (Hughes 1987):

- we compute the transverse shear at the middle of the edges, parallel to the edge

- at the corners, we compute transverse shears in the Cartesian bases, from the strain coming from the 2 edges going to the corners

- we take a mean value from the 3 nodes.

There are no EAS modes in the triangular element. 
The number of integration points is $2 * 2 * 2$ for the quadrangular element and $3 * 2$ for the triangular element.

\section{Numerical integration over the thickness}

The easiest solution is to put several integration points along the thickness. In this case, for a multilayer element, the CPU cost in order to compute the stiffness matrix is proportional to the number of layers and can be large. When we look at the strain and displacement definition, we see that the strain is linear over the thickness (at least for a plate). We put two integration points along the thickness, we compute the strains in these two points. From theses strains, we compute shell axial strain, curvature, shear strain. To make it simple, we show the relation in the $2 \mathrm{D}$ case:

$$
\left|\begin{array}{c}
\varepsilon_{0} \\
\chi \\
\gamma \\
\varepsilon_{z}
\end{array}\right|=\left|\begin{array}{cccccc}
1 / 2 & 0 & 0 & 1 / 2 & 0 & 0 \\
1 / 2 t^{*} & 0 & 0 & -1 / 2 t^{*} & 0 & 0 \\
0 & 0 & 1 / 2 & 0 & 0 & 1 / 2 \\
0 & 1 / 2 & 0 & 0 & 1 / 2 & 0
\end{array}\right| \begin{gathered}
\varepsilon_{x}^{1} \\
\varepsilon_{z}^{1} \\
\gamma^{1} \\
\varepsilon_{x}^{2} \\
\varepsilon_{z}^{2} \\
\gamma^{2}
\end{gathered} \mid \quad \varepsilon_{\text {coque }}=C \varepsilon_{\text {volume }}
$$

From the shell strains, we can compute normal forces, bending moments, shear forces. For instance, for a shell with a linear isotropic material, we have:

$$
\left|\begin{array}{c}
N \\
M \\
T \\
\sigma_{z}
\end{array}\right|=\left|\begin{array}{llll}
E t & & & \\
& E t^{3} / 12 & & \\
& & G t & \\
& & & \alpha^{-2} E
\end{array}\right|\left|\begin{array}{c}
\varepsilon_{0} \\
\chi \\
\gamma \\
\varepsilon_{z}
\end{array}\right| \quad \sigma_{\text {coque }}=H_{\text {coque }} \varepsilon_{\text {coque }}
$$

With $\alpha=L / t$.

In other cases, we perform a numerical integration over the thickness in order to compute the shell stresses. Once we have the shell stresses, we compute the stresses conjugated to the local strains at the two integration points along the thickness:

$$
\left\langle\begin{array}{cc}
\varepsilon_{1}^{T} & \varepsilon_{2}^{T}
\end{array}\right\rangle\left|\begin{array}{l}
\sigma_{1} \\
\sigma_{2}
\end{array}\right| d z=\varepsilon_{\text {coque }}^{T} \sigma_{\text {coque }} \quad\left|\begin{array}{l}
\sigma_{1} \\
\sigma_{2}
\end{array}\right|=\frac{1}{d z} C^{T} \sigma_{\text {coque }}
$$

Similar formulas are used for the local Hooke matrix. With such a procedure, the CPU cost for the stiffness matrix does not depend on the number of layers. The large matrix products are performed with only two integration points over the thickness, even for multilayered element.

As the constitutive equation is written between shell strains and stresses, it is easy to introduce a shear correction factor in the constitutive law as in a classical shell element. For instance, for a monolayer element, we do not used $G t$ in the constitutive equation, we use 5/6 Gt. Similar correction can be used for multilayered element. 


\section{Numerical applications}

The element has been introduced in Samcef ${ }^{\mathrm{TM}}$ software. All the applications are run with the $3^{\text {rd }}$ version of the element.

\subsection{Square plate under pressure}

The plate is simply supported, only a quarter is modelized. The length is taken equal to $400 \mathrm{~mm}$, the thickness $2 \mathrm{~mm}$, Young modulus $2.1 \times 10^{6} \mathrm{MPa}$, Poisson ratio 0.3 , pressure $0.014794 \mathrm{MPa}$. The theoretical displacement (Kirchhoff theory) at the centre of the plate is equal to $1 \mathrm{~mm}$. Table 4 gives the displacement obtained with the quadrangular and triangular elements in function of the number of elements (along one edge). A good and quick convergence is observed, also for the triangular element.

Table 4. Square plate, convergence study

\begin{tabular}{|c|c|c|}
\hline$n$ & Quadrangular élément & Triangular \\
\hline 2 & 0.989 & 0.883 \\
\hline 4 & 0.998 & 0.978 \\
\hline 8 & 1.000 & 0.993 \\
\hline 16 & 1.001 & 0.999 \\
\hline 32 & 1.002 & 1.001 \\
\hline
\end{tabular}

\subsection{Hemispherical shell under concentrated loads}

The problem is described in Figure 2. It is a classical shell problem (Mac Neal et al., 1985), which is sensitive to membrane locking. The reference solution is taken equal to 0.094 , it is not a analytical solution. Table 5 gives the numerical displacement $(* 10000)$ for different meshes.

Table 5. Hemispherical shell, convergence study

\begin{tabular}{|c|c|c|}
\hline$n$ & Quadrangular & Triangular \\
\hline 4 & 979 & 466 \\
\hline 8 & 944 & 850 \\
\hline 16 & 935 & 913 \\
\hline 32 & 935 & 927 \\
\hline
\end{tabular}




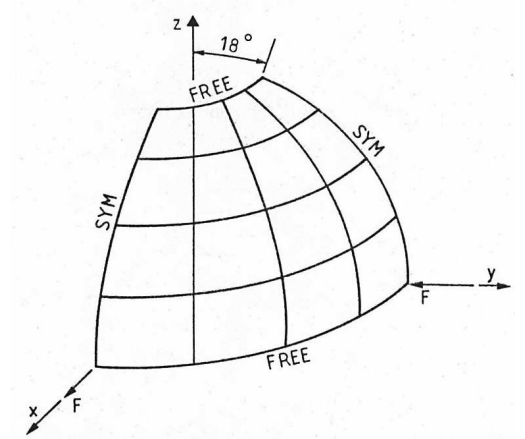

Figure 2. Hemispherical shell under concentrated load. $R=10, E=6.825 \times 10^{7}, v=0.3$, $F=1$, thickness $=0.04$

\subsection{Pinched cylinder with end diaphragms}

The problem is described in Figure 3. It is a classical shell problem (Batoz et al., 1990). The reference displacement is taken equal to $0.18248 \times 10^{-4}$. We compare our results to published one with similar elements (Alves de Souza et al., 2003, AbedMeraim et al., 2007). Table 6 gives the ration between the numerical displacement and the reference one for different regular meshes $(N=$ number of elements on one edge). Symmetry conditions are used.

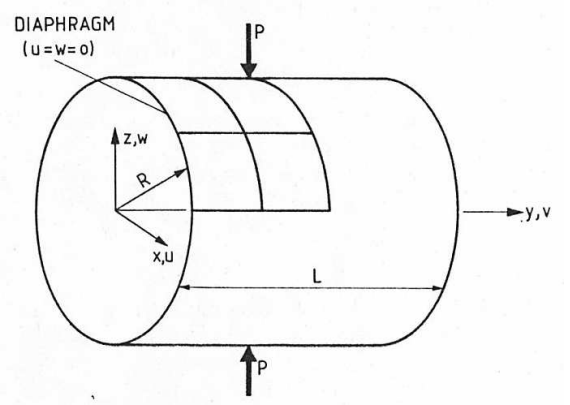

Figure 3. Pinched cylinder with end diaphragms, $R=300, L=600, E=3 \times 10^{6}$, $v=0.3, P=1$, thickness $=3$ 
Table 6. Pinched cylinder, convergence study

\begin{tabular}{|c|c|c|c|c|}
\hline $\boldsymbol{N}$ & Quadrangular & Triangular & Alves de Souza & Abed-Meraim \\
\hline 4 & 0,357 & 0.168 & 0,104 & 0,387 \\
\hline 8 & 0,744 & 0.545 & 0,494 & 0,754 \\
\hline 16 & 0,934 & 0.843 & 0,912 & 0,940 \\
\hline 32 & 0,991 & 0.958 & 0,995 & 0,997 \\
\hline
\end{tabular}

\subsection{Pinched cylinder with end diaphragms, non linear analysis}

This is the same problem as the previous one but a non-linear analysis is performed. Large displacements are taken into account. The Young modulus is taken equal to 3000 . The material is a plastic one, the initial Yield stress is equal to 24.3 and the plastic modulus to 300 (Korelc et al., 1996). A $20 * 20$ element mesh is used, taking into account the symmetry of the problem. Figure 4 shows the isovalues of the equivalent plastic deformation at the end of the analysis and figure 5 shows the applied force in function of the displacement. The results are closed to the reference one (not shown).

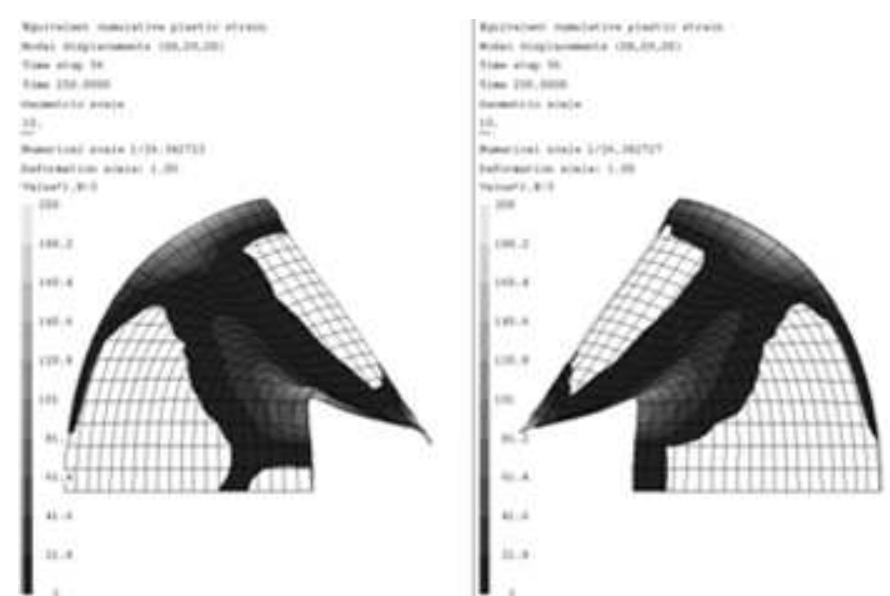

Figure 4. Isovalues of equivalent plastic strain 


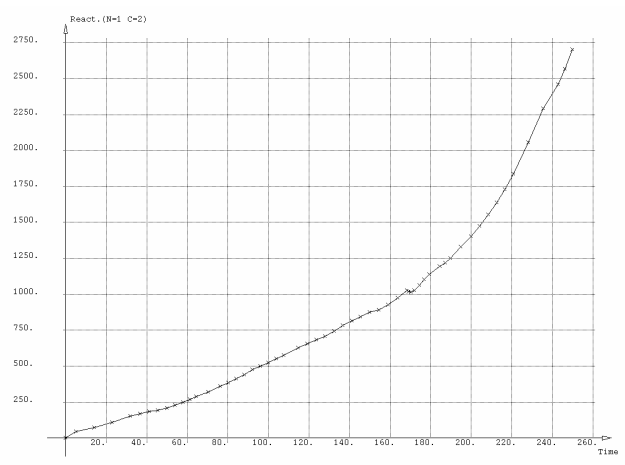

Figure 5. Curve load - displacement

\subsection{Post buckling analysis of a stiffened panel}

The last problem is the post buckling analysis of a stiffened panel. There is contact between the skin and the stringers. First, there is local buckling between the stringers, with a stable post-buckling behaviour. Then, there is buckling of the stringers. Figure 6 shows the isovalues of the transversal displacement,

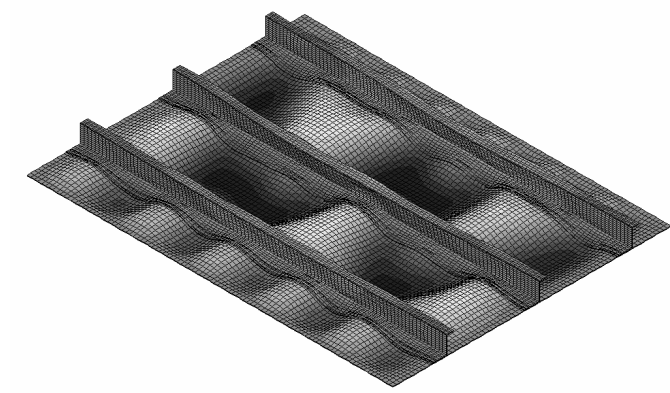

Figure 6. Isovalue of transversal displacement

\section{Conclusion}

The quadrangular and triangular elements give good results on the examples. Their behaviour is good in linear and non-linear analysis. Special care has been taken on the CPU cost for multi-layer case. 


\section{References}

Abed-Meraim F. and Combescure A., "A physically stabilized and locking-free formulation of the (SHB8PS) solid-shell element", Revue Européenne de Mécanique Numérique, vol. $16, \mathrm{n}^{\circ} 8,2007$, p. 1037-1072.

Alves de Sousa R.J., Natal Jorge R.M., Fontes Valente R.A. and César de Sà J.M.A., "A new volumetric and shear locking-free 3D enhanced strain element", Engineering Computations, vol. 20, n 7, 2003, p. 896-925.

Batoz J.L. and Dhatt G, « Modélisation des structures par éléments finis - vol. 3 Coques », Hermès, 1990.

Dvorkin E.N. and Bathe K. J., "A continuum mechanics based four-node shell element for general nonlinear analysis", Engineering Computations, vol. 1, 1984, p. 77-88.

Hughes T.J.R., The Finite Element Method, linear Static and Dynamic finite Element Analysis, Prentice-Hall INC, 1987.

Korelc J. and Wriggers P., "An efficient 3D enhanced strain four node element with Taylor expansion of the shape functions", Computational Mechanics, vol. 19, 1996, p. 30-40.

Mac Neal R.H. and Harder R.L., "A proposed standard set of problems to test finite element accuracy”, Finite Elements in Analysis and Design, 1, 1985, p. 3-20.

Simo J.C., Armero F. and Taylor R.L., "Improved versions of assumed enhanced strain trilinear element for 3D finite deformation problem", Comput. Methods Appl. Mech Engng, vol. 110,1993 , p. 359-386. 
